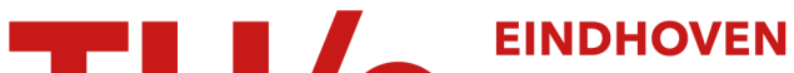 \\ UNIVERSITY OF \\ TECHNOLOGY
}

\section{Synthesis of distributed robust H-Infinity controllers for interconnected discrete time systems}

Citation for published version (APA):

Horssen, E. P. V., \& Weiland, S. (2016). Synthesis of distributed robust $\mathrm{H}$-Infinity controllers for interconnected discrete time systems. IEEE Transactions on Control of Network Systems, 3(3), 286-295.

https://doi.org/10.1109/TCNS.2015.2476199

DOI:

10.1109/TCNS.2015.2476199

Document status and date:

Published: 01/09/2016

Document Version:

Accepted manuscript including changes made at the peer-review stage

Please check the document version of this publication:

- A submitted manuscript is the version of the article upon submission and before peer-review. There can be important differences between the submitted version and the official published version of record. People interested in the research are advised to contact the author for the final version of the publication, or visit the $\mathrm{DOI}$ to the publisher's website.

- The final author version and the galley proof are versions of the publication after peer review.

- The final published version features the final layout of the paper including the volume, issue and page numbers.

Link to publication

\section{General rights}

Copyright and moral rights for the publications made accessible in the public portal are retained by the authors and/or other copyright owners and it is a condition of accessing publications that users recognise and abide by the legal requirements associated with these rights.

- Users may download and print one copy of any publication from the public portal for the purpose of private study or research.

- You may not further distribute the material or use it for any profit-making activity or commercial gain

- You may freely distribute the URL identifying the publication in the public portal.

If the publication is distributed under the terms of Article $25 \mathrm{fa}$ of the Dutch Copyright Act, indicated by the "Taverne" license above, please follow below link for the End User Agreement:

www.tue.nl/taverne

Take down policy

If you believe that this document breaches copyright please contact us at:

openaccess@tue.nl

providing details and we will investigate your claim. 


\title{
Synthesis of Distributed Robust H-infinity Controllers for Interconnected Discrete Time Systems
}

\author{
E.P. van Horssen \\ S. Weiland
}

\begin{abstract}
This paper presents an algorithm for the synthesis of robust distributed controllers for interconnected linear discrete time systems. For a network of interconnected uncertain linear time-invariant systems, the distributed controller achieves robust stability and a guaranteed level of robust performance in a welldefined $\mathcal{H}_{\infty}$ sense. The setting of the paper is in discrete time. Based on the theory of dissipative dynamical systems, conditions for the analysis of robust stability and robust performance of networks are derived in terms of feasibility tests of linear matrix inequalities. From these conditions, computationally tractable synthesis conditions are derived. An iterative D-K type of synthesis algorithm is proposed that yields a robust distributed controller. Convergence properties of the algorithm are inferred.
\end{abstract}

Keywords-Controller synthesis, distributed control, discrete time systems, interconnected systems, linear matrix inequalities (LMIs), robustness, robust stability.

\section{INTRODUCTION}

$\mathbf{T}$ HE design of distributed controllers that achieve guarantees for the reliable operation and stability of networks of dynamical systems leads to a broad range of technical and scientific challenges in the control community. A distributed control architecture allows multiple controllers to exchange information in a well-defined manner so as to accomplish a desirable behavior of the network. This provides advantages over centralized control, for which communication architectures may be impractical, infeasible or incomputable, and over decentralized control, which may not provide desired global stability or robustness properties of an interconnected system.

The focus of this paper is on the synthesis of robust distributed controllers for networks of interconnected linear discrete time systems with uncertainty. We propose a computationally tractable algorithm for the synthesis of such controllers that achieve robust stability and robust performance in a well-defined $\mathcal{H}_{\infty}$ sense. The main results provide LMI-based algorithms for the synthesis of such controllers.

This paper builds on earlier work in a similar context. Specifically, this paper is inspired by the settings of [1][3] in which LMI-based tools have been developed for the synthesis of distributed controllers achieving a bounded $\mathcal{H}_{\infty}$ performance for networks of systems over an arbitrary graph.

E.P. van Horssen and S. Weiland are with the Control Systems Technology Group of the Department of Mechanical Engineering and the Control Systems Group of the Department of Electrical Engineering, respectively, at Eindhoven University of Technology, P.O. Box 513, 5600 MB Eindhoven, The Netherlands. Email: e.p.v.horssen@tue.nl, s.weilandetue.nl
The generalized notion of dissipativity, initiated by Willems in [4], has been at the basis of this development and led to extensions in [5] to incorporate the possibility of passive interconnections. More recently, these ideas have led to generalization to incorporate uncertainty in the distributed control paradigm with concrete LMI-based synthesis algorithms for robust distributed control presented in [6], [7]. These algorithms apply to networks of continuous time linear time-invariant dynamical systems that are subject to model uncertainty.

In [8], robustness against communication delays was studied. These contributions focus on continuous time systems. A first study on the stability of networks of discrete time systems has been made in [9]. Distributed $\mathcal{H}_{\infty}$ control of spatially interconnected systems with random communication packet losses was also studied in [10], but not from a dissipation perspective. In [11], the authors employ a gain scheduling approach to the distributed control of LPV systems, in which temporal and spatial variations of parameters are explicitly distinguished and viewed as parametric uncertainties of different types.

In view of the need for computationally tractable algorithms that synthesize robust distributed controllers, we believe that there is a demand for generalizing the results in [1]-[3], [6], [7] to networks of discrete time systems. This is the purpose of the present paper. In particular, the study of discrete time systems allows to explicitly incorporate network delays in the models by augmenting state vectors. Novel analysis and synthesis results are derived for networks of discrete time systems and we address the specific technical problems with networks of uncertain discrete time systems. This paper contributes with a synthesis procedure for distributed systems in which robustness against model uncertainty is taken into account and in which robust stability and robust $\mathcal{H}_{\infty}$ performance is guaranteed for the controlled network. A computationally tractable synthesis algorithm is presented. A control example for an electrical power network shows the feasibility of the synthesis procedure.

This paper is organized as follows. In Section II, the properties and structure of a distributed system and controller are explained and some analysis tools are presented that are used to formulate the main problems in Section III. Section IV presents a number of analysis results which provide sufficient conditions for robust stability and performance of a distributed system. From these theorems, computationally tractable synthesis results are obtained in Section V, and an algorithm is presented to synthesize a robust distributed controller. Simulations are presented and discussed in Section VI. Conclusions and comments on future work are given in Section VII. 


\section{A. Notation}

Let $\mathbb{R}$ and $\mathbb{N}$ denote the set of real numbers and the set of natural numbers or positive integers, respectively. The set of non-negative integers is denoted by $\mathbb{N}_{0}$. The set of $n \times m$ real matrices is denoted by $\mathbb{R}^{n \times m}$ and the set of $n \times n$ real symmetric matrices by $\mathbb{R}_{S}^{n}$. The notation $\mathbb{N}_{\geq c_{1}}$ and $\mathbb{N}_{\left(c_{1}, c_{2}\right]}$ is used to denote the sets $\left\{k \in \mathbb{N}_{0} \mid \bar{k} \geq c_{1}\right\}$ and $\left\{k \in \mathbb{N}_{0} \mid c_{1}<k \leq c_{2}\right\}$, respectively, for some $c_{1}, c_{2} \in \mathbb{N}_{0}$. The cardinality of a finite set $\mathcal{V}$ is denoted by $\operatorname{card}(\mathcal{V})$. For matrices $A, B \in \mathbb{R}_{S}^{n}$, the inequality $A \prec B$ (respectively, $A \preceq B$ ) means that $B-A$ is symmetric positive definite (positive semi-definite, respectively). Transposition of vectors and matrices is denoted by the superscript $T$. The operator $\operatorname{col}(\cdot)$ stacks its arguments in a column vector. The block-diagonal matrix that has matrices $A_{k}, \ldots A_{l}$ on its blockdiagonal entries is denoted by $\operatorname{diag}_{i \in \mathbb{N}_{[k, l]}} A_{i}$. The Hölder $p$ norm of a vector $x$ is denoted by $\|x\|_{p}$ for $p \in \mathbb{N}_{[1, \infty]}$. The inertia of a matrix $M \in \mathbb{R}_{S}^{n}$ is denoted by $\operatorname{in}(M): \mathbb{R}_{S}^{n} \rightarrow \mathbb{R}_{\geq 0}^{3}$ and is defined as the triplet $i n(M):=\left(a_{-}, a_{0}, a_{+}\right)$of negative, zero and positive eigenvalues of $M$, respectively. Finally, for $n \in \mathbb{N}$, the space of $n$-dimensional vector-valued infinite sequences that are square summable is defined as:

$$
\ell_{2}^{n}:=\left\{x: \mathbb{N}_{[0, \infty]} \rightarrow \mathbb{R}^{n} \quad \mid \quad\|x\|_{2}^{2}:=\sum_{i=0}^{\infty}\|x(i)\|_{2}^{2}<\infty\right\} .
$$

The extended space of $n$-dimensional vector-valued infinite sequences is defined $\forall n \in \mathbb{N}$ as $\ell_{2 e}^{n}:=\left\{x: \mathbb{N}_{[0, \infty]} \rightarrow \mathbb{R}^{n}\right\}$. Note that $\ell_{2}^{n} \subset \ell_{2 e}^{n}$.

\section{DISCRETE TIME DISTRIBUTED SYSTEMS}

Inspired by the setting of distributed control synthesis in [2], [5], [7] we view a distributed system as a graph in which $L$ arbitrary dynamical systems are interconnected. Here, we focus on interconnections of discrete time, linear time-invariant and uncertain dynamical systems. This section presents the structure and properties of such distributed systems.

\section{A. Distributed system definition}

The structure of an arbitrary distributed system $H$ is represented by a graph $\mathcal{G}_{H}:=\left(\mathcal{V}_{H}, \mathcal{E}_{H}\right)$, where $\mathcal{V}_{H}$ is the set of vertices, and $\mathcal{E}_{H} \subseteq \mathcal{V}_{H} \times \mathcal{V}_{H}$ is the set of edges between the vertices [12]. For a distributed system $H$ consisting of $L$ subsystems, the set of vertices $\mathcal{V}_{H}$ is identified with a set of dynamical systems $\left\{H^{1}, \ldots, H^{L}\right\}$ and the set of non-oriented edges $\mathcal{E}_{H}$ is defined by the set of pairs $\left\{\left(H^{i}, H^{j}\right) \mid i \leq j, \quad i, j \in \mathbb{N}_{[1, L]}\right\}$ where $\left(H^{i}, H^{j}\right) \in \mathcal{E}_{H}$ if and only if $\left(H^{i}, H^{j}\right)$ are interconnected. The neighbors of the $i$ th subsystem are defined by the index set $\mathcal{N}^{i}=\{j \in$ $\left.\mathbb{N}_{[1, L]} \mid\left(H^{i}, H^{j}\right) \in \mathcal{E}_{H}\right\}$. Note that a subsystem can be selfconnected in this definition.

The focus of this paper is on uncertain distributed systems $G_{\Delta}$ in which the graph $\mathcal{G}_{G_{\Delta}}=\left(\mathcal{V}_{G_{\Delta}}, \mathcal{E}_{G_{\Delta}}\right)$ has vertices $\left\{G_{\Delta}^{1}, \ldots, G_{\Delta}^{L}\right\}$ that represent uncertain dynamical systems (referred to as subsystems) where the uncertainty admits a welldefined linear-fractional representation, as defined in the next subsection.

\section{B. Subsystem representation}

The $i$ th subsystem $G_{\Delta}^{i}$ in the distributed system $G_{\Delta}$ consists of an interconnection of a nominal multi-channel linear time-invariant (LTI) system $G_{0}^{i} \quad: \quad \mathbb{R}^{n_{G}^{i}+n_{\Delta}^{i}+n_{d}^{i}+n_{u}^{i}} \rightarrow \mathbb{R}^{n_{G}^{i}+n_{\Delta}^{i}+n_{z}^{i}+n_{y}^{i}}$ and an LTI system $\Delta^{i}: \ell_{2}^{n_{\Delta}^{i}} \rightarrow \ell_{2}^{n_{\Delta}^{i}}$, that is assumed to belong to a class of stable dynamic LTI systems $\boldsymbol{\Delta}^{i}$, representing the uncertainty. Specifically, for all $i=1, \ldots, L$, the dynamics of the uncertain discrete time LTI subsystem $G_{\Delta}^{i}$ is represented by

$$
\begin{gathered}
p^{i}(k)=\Delta^{i}\left(q^{i}(k)\right) \text { for some } \Delta^{i} \in \Delta^{i} \\
{\left[\begin{array}{c}
x^{i}(k+1) \\
w^{i}(k) \\
q^{i}(k) \\
z^{i}(k) \\
y^{i}(k)
\end{array}\right]=\left[\begin{array}{lllll}
A_{x x}^{i} & A_{x v}^{i} & B_{x p}^{i} & B_{x d}^{i} & B_{x u}^{i} \\
A_{w x}^{i} & A_{w v}^{i} & B_{w p}^{i} & B_{w d}^{i} & B_{w u}^{i} \\
C_{q x}^{i} & C_{q v}^{i} & D_{q p}^{i} & D_{q d}^{i} & D_{q u}^{i} \\
C_{z x}^{i} & C_{z v}^{i} & D_{z p}^{i} & D_{z d}^{i} & D_{z u}^{i} \\
C_{y x}^{i} & C_{y v}^{i} & D_{y p}^{i} & D_{y d}^{i} & D_{y u}^{i}
\end{array}\right]\left[\begin{array}{c}
x^{i}(k) \\
v^{i}(k) \\
p^{i}(k) \\
d^{i}(k) \\
u^{i}(k)
\end{array}\right]}
\end{gathered}
$$

and we group related signals as channels of (inputs, outputs) as

$$
\begin{array}{llll}
\text { interconnection channel } & \left(v^{i}, w^{i}\right) \in \mathbb{R}^{2 n_{G}^{i}}, \\
\text { uncertainty channel } & \left(p^{i}, q^{i}\right) \in \mathbb{R}^{2 n_{\Delta}^{i}}, \\
\text { performance channel } & \left(d^{i}, z^{i}\right) \in \mathbb{R}_{d}^{n_{d}^{i}+n_{z}^{i}}, \\
\text { control channel } & \left(u^{i}, y^{i}\right) \in \mathbb{R}^{n_{u}^{i}+n_{y}^{i}} .
\end{array}
$$

Here, $x^{i}(k) \in \mathbb{R}^{m_{G}^{i}}$ with $m_{G}^{i}=m^{i} \in \mathbb{N}$ is the state variable. The nominal state space representation (2) is denoted by $G_{0}^{i}$ and we remark that the uncertain subsystem $G_{\Delta}^{i}$ is a linear fractional representation of the nominal system $G_{0}^{i}$ with the operator $\Delta^{i}$. The set $\Delta^{i}$ of stable LTI systems is assumed to be known.

If $G_{\Delta}^{i}$ is interconnected with $G_{\Delta}^{j}$, the interconnection channel $\left(v^{i}, w^{i}\right)$ of subsystem $i$ is further partitioned, as $v^{i}=\operatorname{col}\left(v^{i 1}, \ldots, v^{i L}\right)$ and $w^{i}=\operatorname{col}\left(w^{i 1}, \ldots, w^{i L}\right)$, such that $\left(v^{i j}, w^{i j}\right) \in \mathbb{R}^{2 n_{G}^{i j}}$ denotes the interconnection channel between subsystem $i$ and $j$. To simplify the analysis, the matrix $A_{w v}^{i}$ is forced to be square by constraining $w^{i j}$ and $v^{i j}$ to share the same dimension, say $n_{G}^{i j}$. This can always be achieved, possibly after adding zero rows or zero columns to $A_{w v}^{i}$. Without loss of generality, we assume there are no selfconnections, since any such connection can be incorporated in $A_{x x}^{i}$.

The uncertainty channel $\left(p^{i}, q^{i}\right)$ with $p^{i}, q^{i} \in \mathbb{R}^{n_{\Delta}^{i}}$ perturbs the system through the operator $\Delta^{i}$. The performance channel $\left(d^{i}, z^{i}\right)$ consist of external disturbance inputs $d^{i} \in \mathbb{R}^{n_{d}^{i}}$ and performance outputs $z^{i} \in \mathbb{R}^{n_{z}^{i}}$. The control channel $\left(u^{i}, y^{i}\right)$ connects the system to a controller with control input $u^{i} \in \mathbb{R}^{n_{u}^{i}}$ and measurement output $y^{i} \in \mathbb{R}^{n_{y}^{i}}$ When the control channels of the subsystems are not considered, we refer to the distributed system $G_{\Delta}$ as the open loop system. Throughout the paper, we also refer to (1)-(2) as the open loop or uncontrolled system.

\section{Controller representation}

A distributed controller $K$ for a distributed system $G_{\Delta}$ is represented by a graph $\mathcal{G}_{K}=\left(\mathcal{V}_{K}, \mathcal{E}_{K}\right)$, where we identify the vertices $\mathcal{V}_{K}$ with the set of local controllers 
$\left\{K^{1}, \ldots, K^{L}\right\}$ and the edges $\mathcal{E}_{K}$ with the interconnections between the controllers. More specifically, we assume $\operatorname{card}\left(\mathcal{V}_{K}\right)=\operatorname{card}\left(\mathcal{V}_{G_{\Delta}}\right)$ and only allow connections between controllers if their respective plants are also connected, i.e. a collocated controller. This is formalized by requiring that edges $\left(K^{i}, K^{j}\right) \in \mathcal{E}_{K}$ if and only if edges $\left(G_{\Delta}^{i}, G_{\Delta}^{j}\right) \in \mathcal{E}_{G_{\Delta}}$. For $i=1, \ldots, L$, a local controller $K^{i}: \mathbb{R}^{n_{K}^{i}+n_{y}^{i}} \rightarrow \mathbb{R}^{n_{K}^{i}+n_{u}^{i}}$ is an LTI system that is represented in state space form by

$$
\left[\begin{array}{c}
x_{K}^{i}(k+1) \\
w_{K}^{i}(k) \\
u^{i}(k)
\end{array}\right]=\left[\begin{array}{lll}
\left(A_{x x}^{i}\right)_{K} & \left(A_{x v}^{i}\right)_{K} & \left(B_{x y}^{i}\right)_{K} \\
\left(A_{w x}^{i}\right)_{K} & \left(A_{w v}^{i}\right)_{K} & \left(B_{w y}^{i}\right)_{K} \\
\left(C_{u x}^{i}\right)_{K} & \left(C_{u v}^{i}\right)_{K} & \left(D_{u y}^{i}\right)_{K}
\end{array}\right]\left[\begin{array}{c}
x_{K}^{i}(k) \\
v_{K}^{i}(k) \\
y^{i}(k)
\end{array}\right] .
$$

Here, $x_{K}^{i} \in \mathbb{R}^{m_{K}^{i}}$ with $m_{K}^{i}=m_{G}^{i}=m^{i}$ denotes the state variable, $\left(v_{K}^{i}, w_{K}^{i}\right) \in \mathbb{R}^{2 n_{K}^{i}}$ is the controller interconnection channel, and $\left(u^{i}, y^{i}\right) \in \mathbb{R}^{n_{u}^{i}+n_{y}^{i}}$ is the control channel of the $i$ th controller. Conform the partitioning of the interconnection channels in the subsystems of $G_{\Delta}$, the controller interconnection channel is further partitioned into $\left(v_{K}^{i j}, w_{K}^{i j}\right) \in \mathbb{R}^{2 n_{K}^{i j}}$ for any pair $(i, j)$ for which $\left(K^{i}, K^{j}\right) \in \mathcal{E}_{K}$.

\section{Controlled system}

The interconnection of the uncertain distributed system $G_{\Delta}$ and the distributed controller $K$ defines the controlled system or closed loop system $\left(G_{\Delta}\right)_{C}: d:=$ $\operatorname{col}\left(d^{1}, \ldots, d^{L}\right) \rightarrow z:=\operatorname{col}\left(z^{1}, \ldots, z^{L}\right)$ with graph $\mathcal{G}_{\left(G_{\Delta}\right)_{C}}=\left(\mathcal{V}_{\left(G_{\Delta}\right)_{C}}, \mathcal{E}_{\left(G_{\Delta}\right)_{C}}\right)$. The vertices $\mathcal{V}_{\left(G_{\Delta}\right)_{C}}$ correspond to the set $\left\{\left(G_{\Delta}^{i}\right)_{C}:=S\left(G_{\Delta}^{i}, K^{i}\right), i=1, \ldots, L\right\}$, where $S(\cdot, \cdot)$ denotes the Redheffer star product [13]. It follows that edges $\left(\left(G_{\Delta}^{i}\right)_{C},\left(G_{\Delta}^{j}\right)_{C}\right) \in \mathcal{E}_{\left(G_{\Delta}\right)_{C}}$ if and only if $\left(G_{\Delta}^{i}, G_{\Delta}^{j}\right) \in \mathcal{E}_{G_{\Delta}}$ if and only if $\left(K^{i}, K^{j}\right) \in \mathcal{E}_{K}$.

Given a distributed controller, a local interconnection of a subsystem and its corresponding controller $\left(G_{\Delta}^{i}\right)_{C}$ at node $i$, admits the following representation

$$
\begin{aligned}
p^{i}(k) & =\Delta^{i}\left(q^{i}(k)\right) \text { for some } \Delta^{i} \in \Delta^{i} \\
{\left[\begin{array}{c}
x_{C}^{i}(k+1) \\
w_{C}^{i}(k) \\
q^{i}(k) \\
z^{i}(k)
\end{array}\right] } & =\left(G_{0}^{i}\right)_{C}\left[\begin{array}{l}
x_{C}^{i}(k) \\
v_{C}^{i}(k) \\
p^{i}(k) \\
d^{i}(k)
\end{array}\right]
\end{aligned}
$$

with

$$
\left(G_{0}^{i}\right)_{C}=\left[\begin{array}{llll}
\left(A_{x x}^{i}\right)_{C} & \left(A_{x v}^{i}\right)_{C} & \left(B_{x p}^{i}\right)_{C} & \left(B_{x d}^{i}\right)_{C} \\
\left(A_{w x}^{i}\right)_{C} & \left(A_{w v}^{i}\right)_{C} & \left(B_{w p}^{i}\right)_{C} & \left(B_{w d}^{i}\right)_{C} \\
\left(C_{q x}^{i}\right)_{C} & \left(C_{q v}^{i}\right)_{C} & \left(D_{q p}^{i}\right)_{C} & \left(D_{q d}^{i}\right)_{C} \\
\left(C_{z x}^{i}\right)_{C} & \left(C_{z v}^{i}\right)_{C} & \left(D_{z p}^{i}\right)_{C} & \left(D_{z d}^{i}\right)_{C}
\end{array}\right]
$$

where the closed loop states and interconnection signals are now the stacked states and interconnection signals of the $i$ th subsystem and the $i$ th controller. Hence, $x_{C}^{i}=\operatorname{col}\left(x^{i}, x_{K}^{i}\right) \in$ $\mathbb{R}^{2 m^{i}}, v_{C}^{i}=\operatorname{col}\left(v^{i}, v_{K}^{i}\right) \in \mathbb{R}^{n_{C}^{i}}$ and $w_{C}^{i}=\operatorname{col}\left(w^{i}, w_{K}^{i}\right) \in$ $\mathbb{R}^{n_{C}^{i}}, n_{C}^{i}=n_{G}^{i}+n_{K}^{i}$.

We will extensively exploit the property that the nominal controlled system $\left(G_{0}^{i}\right)_{C}$ can be written as an affine function of the controller parameters $\Theta^{i}$ in (3), provided that $D_{y u}^{i}=0$. Indeed, for $D_{y u}^{i}=0$,

$$
\left(G_{0}^{i}\right)_{C}=\Pi^{i}+\left(\Psi^{i}\right)^{\top} \Theta^{i} \Phi^{i}
$$

with

$$
\begin{aligned}
& \Pi^{i}:=\left[\begin{array}{cccccc}
A_{x x}^{i} & 0 & A_{x v}^{i} & 0 & B_{x p}^{i} & B_{x d}^{i} \\
0 & 0 & 0 & 0 & 0 & 0 \\
A_{w x}^{i} & 0 & A_{w v}^{i} & 0 & B_{w p}^{i} & B_{w d}^{i} \\
0 & 0 & 0 & 0 & 0 & 0 \\
C_{q x}^{i} & 0 & C_{q v}^{i} & 0 & D_{q p}^{i} & D_{q d}^{i} \\
C_{z x}^{i} & 0 & C_{z v}^{i} & 0 & D_{z p}^{i} & D_{z d}^{i}
\end{array}\right] \\
& \left(\Psi^{i}\right)^{\top}:=\left[\begin{array}{ccc}
0 & 0 & B_{x u}^{i} \\
I & 0 & 0 \\
0 & 0 & B_{w u}^{i} \\
0 & I & 0 \\
0 & 0 & D_{q u}^{i} \\
0 & 0 & D_{z u}^{i}
\end{array}\right] \\
& \Theta^{i}:=\left[\begin{array}{lll}
\left(A_{x x}^{i}\right)_{K} & \left(A_{x v}^{i}\right)_{K} & \left(B_{x y}^{i}\right)_{K} \\
\left(A_{w x}^{i}\right)_{K} & \left(A_{w v}^{i}\right)_{K} & \left(B_{w y}^{i}\right)_{K} \\
\left(C_{u x}^{i}\right)_{K} & \left(C_{u v}^{i}\right)_{K} & \left(D_{u y}^{i}\right)_{K}
\end{array}\right], \\
& \Phi^{i}:=\left[\begin{array}{cccccc}
0 & I & 0 & 0 & 0 & 0 \\
0 & 0 & 0 & I & 0 & 0 \\
C_{y x}^{i} & 0 & C_{y v}^{i} & 0 & D_{y p}^{i} & D_{y d}^{i}
\end{array}\right] .
\end{aligned}
$$

\section{E. Interconnections}

The input and output signals that define the interconnection channel $(v, w)$ are restricted to satisfy the constraint:

$$
\left[\begin{array}{c}
w^{i j}(k) \\
v^{i j}(k)
\end{array}\right]=\left[\begin{array}{c}
v^{j i}(k) \\
w^{j i}(k)
\end{array}\right] \quad \forall i \geq j, \forall k \geq 0 .
$$

This algebraic constraint reflects that an interconnection signal $w^{i j}(k)$ which leaves subsystem $i$ enters subsystem $j$. This constraint holds for the subsystems as well as for the local controllers.

Well-posedness of the interconnected system is an important requirement. Following the ideas in [2], sufficient conditions for well-posedness of the interconnected system can be derived in terms of a separability property of properly defined subspaces. These conditions [2, Section IV.C] can be generalized to the present model as was done by [6, Section II.B]. A sufficient condition for the well-posedness of all channels of the system, apart from the controller interconnections, is that, for $i=1, \ldots, L$,

$$
B_{w p}^{i}=0, C_{y v}^{i}=0 \text { or } B_{w u}^{i}=0, D_{y p}^{i}=0, D_{y u}^{i}=0 .
$$

Conditions (12) are rather mild and can be enforced, for example, by placing low-pass filters on the appropriate channels [2], [6].

Consider the matrix $\left(A_{w v}\right)_{K}:=\operatorname{diag}_{i \in \mathbb{N}_{[1, L]}}\left(A_{w v}^{i}\right)_{K}$ and a row-permuting matrix $W_{K}$ representing the interconnections (11) for $v_{K}=\operatorname{col}\left(v_{K}^{1}, \ldots, v_{K}^{L}\right)$ and $w_{K}=\operatorname{col}\left(w_{K}^{1}, \ldots, w_{K}^{L}\right)$ such that $v_{K}=W_{K} w_{K}$. Then, the controller is well-posed if and only if

$$
\operatorname{det}\left(I-W_{K}\left(A_{w v}\right)_{K}\right) \neq 0
$$

holds. Moreover, given the conditions (12), we have that the closed loop system is well-posed if and only if the controller is well-posed. 


\section{F. Stability}

We use the notion of Lyapunov stability [14, Theorem 2.2.4] to prove global exponential stability (GES) of a distributed system. Given that a system is well-posed, robust stability is defined as follows.

Definition 1 (Robust stability). The (controlled) system with uncertainties taken from $\boldsymbol{\Delta}$, satisfying (1)-(2) or (4)-(5), is called robustly stable if it is well-posed and, for all $\Delta \in \Delta$ and for all initial conditions and for any (disturbance) input in the class $\ell_{2}$, the state and all outputs belong to $\ell_{2}$.

\section{G. Performance}

The robust performance bound for the uncertain but robustly stable system $G_{\Delta}: d \in \ell_{2 e}^{n_{d}} \rightarrow z \in \ell_{2 e}^{n_{z}}$, such as (1)-(2) or (4)(5), is defined as follows.

Definition 2 (Robust performance). The (controlled) uncertain system $G_{\Delta}$ achieves robust performance of level $\gamma$ if it is wellposed, robustly stable and, with initial state set to zero, satisfies

$$
\left\|G_{\Delta}\right\|_{2,2}:=\sup _{d \neq 0, d \in \ell_{2}^{n_{d}}, \Delta \in \Delta} \frac{\|z\|_{2}}{\|d\|_{2}}<\gamma .
$$

Note that robust performance therefore yields a guaranteed bound on the worst case gain, measured in $\ell_{2}$ sense, from (disturbance) input $d$ to (performance) output $z$ in view of all possible uncertainties that affect the system. Since for any stable LTI system $G$ it holds that $\|G\|_{2,2}=$ $\sup _{\theta \in[0, \pi]} \sigma_{\max }\left(G\left(e^{j \theta}\right)\right)=:\|G\|_{\infty}$ we therefore achieve performance in $\mathcal{H}_{\infty}$ sense.

\section{PROBLEM FORMULATION}

In this section we formalize the main problems that will be considered and solved in this paper. The first problem relates to analysis of a system as described in Section II which can be either an open or closed loop system.

Problem 1 (Analysis). Given an uncertain distributed system $G_{\Delta}$ defined as in Section II, find computationally verifiable conditions to test whether the open loop uncertain distributed system $G_{\Delta}$ is well-posed, robustly stable and achieves robust performance of level $\gamma>0$.

The second problem addresses the synthesis of distributed controllers as described in Section II-C.

Problem 2 (Synthesis). Given an uncertain distributed system $G_{\Delta}$ defined as in Section II, find a computationally tractable synthesis procedure that generates a distributed controller $K$, with structure as given in Section II-C, that renders the controlled uncertain distributed system $\left(G_{\Delta}\right)_{C}$, as defined in Section II-D, well-posed, robustly stable while achieving robust performance of level $\gamma$.

\section{ANALYSIS}

\section{A. Dissipation of discrete time systems}

For the analysis of stability and performance of an uncertain distributed system, we make use of concepts from the theory of dissipative systems initiated by [4]. This will yield a natural and methodologically elegant setting to study properties of interconnected dynamical systems and eventually leads to computationally efficient tools [15].

Definition 3 (Discrete time dissipativity). A nominal discrete time subsystem $G_{0}^{i}$ as in (2) is dissipative with respect to a supply function $\Phi^{i}(\cdot)$, depending on all input and output signals of the system, if there exists a storage function $V^{i}: \mathbb{R}^{m_{G}^{i}} \rightarrow \mathbb{R}$, depending on the state of the system, such that, for all integers $M \geq 0$,

$$
V^{i}\left(x^{i}(M)\right)-V^{i}\left(x^{i}(0)\right) \leq \sum_{k=0}^{M-1} \Phi^{i}(k)
$$

holds for all possible system trajectories generated by (1)-(2). Here, for notational brevity the signal variables are omitted and $\Phi^{i}(k)$ represents the supply delivered to the system at time $k$, viewed as function of the signal variables at time instance $k$. The system is said to be strictly dissipative if the inequality in (15) is a strict one.

Hence, a dissipative system is characterized by the property that the increase of energy that is stored in the subsystem cannot exceed the energy supplied to the system over any time horizon $[0, M)$.

For the nominal system $G_{0}^{i}$ we consider aggregated supply functions $\Phi^{i}(k)$ of the form

$$
\Phi^{i}(k):=P^{i}(k)+U^{i}(k)+S^{i}(k)
$$

where

$$
\begin{aligned}
P^{i}\left(v^{i}, w^{i}\right) & :=\sum_{j} P^{i j}\left(v^{i j}, w^{i j}\right), \\
P^{i j}\left(v^{i j}, w^{i j}\right) & :=\left[\begin{array}{c}
w^{i j} \\
v^{i j}
\end{array}\right]^{\top} X^{i j}\left[\begin{array}{l}
w^{i j} \\
v^{i j}
\end{array}\right], \\
U^{i}\left(p^{i}, q^{i}\right) & :=-\left[\begin{array}{l}
q^{i} \\
p^{i}
\end{array}\right]^{\top} D_{\Delta}^{i}\left[\begin{array}{c}
q^{i} \\
p^{i}
\end{array}\right], \\
S^{i}\left(d^{i}, z^{i}\right) & :=\gamma\left\|d^{i}\right\|^{2}-\frac{1}{\gamma}\left\|z^{i}\right\|^{2} \\
& =\left[\begin{array}{c}
z^{i} \\
d^{i}
\end{array}\right]^{\top}\left[\begin{array}{cc}
-\frac{1}{\gamma} I & 0 \\
0 & \gamma I
\end{array}\right]\left[\begin{array}{l}
z^{i} \\
d^{i}
\end{array}\right],
\end{aligned}
$$

with $\gamma>0, X^{i j} \in \mathbb{R}_{S}^{2 n_{G}^{i j}}$ and $D_{\Delta}^{i} \in \mathbb{R}_{S}^{2 n_{\Delta}^{i}}$. These define quadratic supply functions on the interconnection, uncertainty and performance channels, respectively. The supply functions represent the intuitive idea on how the nominal system exchanges power with its environment through its different channels. Here, for the time being, the control channel is disregarded. In (16) all variables have dimensions that match the dimensions of the time-varying signals with the same name in (2). We consider storage functions

$$
V^{i}\left(x^{i}\right):=\left(x^{i}\right)^{\top} X_{T}^{i} x^{i}
$$

that are quadratic in $x^{i}$ where $X_{T}^{i} \in \mathbb{R}_{S}^{m^{i}}$. 
The matrix $D_{\Delta}^{i} \in \mathbb{R}_{S}^{2 n_{\Delta}^{i}}$ in (18) is assumed to be partitioned accordingly with the uncertainty channel as

$$
D_{\Delta}^{i}=\left[\begin{array}{cc}
D_{11}^{i} & D_{12}^{i} \\
\left(D_{12}^{i}\right)^{\top} & D_{22}^{i}
\end{array}\right] .
$$

\section{B. Interconnection neutrality}

If subsystem $G_{\Delta}^{i}$ is dissipative with respect to supply function $P^{i j}$ and its neighboring subsystem $G_{\Delta}^{j}$ is dissipative with respect to supply function $P^{j i}$, then the interconnection channel $(v, w)$ satisfies (11) together with the neutrality condition

$$
P^{i j}\left(v^{i j}, w^{i j}\right)+P^{j i}\left(v^{j i}, w^{j i}\right)=0,
$$

that reflects that no power is lost nor injected in the interconnection channel [15]. Using the combination of (17) and (11) it is easily shown that the neutrality condition (22) is equivalent to the following algebraic requirement on $X^{i j}$ and $X^{j i}$ :

$$
X^{i j}=-\left[\begin{array}{cc}
0 & I_{n_{G}^{i j}} \\
I_{n_{G}^{i j}} & 0
\end{array}\right] X^{j i}\left[\begin{array}{cc}
0 & I_{n_{G}^{i j}} \\
I_{n_{G}^{i j}} & 0
\end{array}\right]
$$

which is required for all $i$ and $j$.

This has implications for the matrices $X^{i j}$. Indeed, if $X^{i j}$ is partitioned accordingly with the signals $\left(v^{i j}, w^{i j}\right)$ into four $n_{G}^{i j} \times n_{G}^{i j}$ blocks as in

$$
X^{i j}:=\left[\begin{array}{cc}
X_{11}^{i j} & X_{12}^{i j} \\
\left(X_{12}^{i j}\right)^{\top} & X_{22}^{i j}
\end{array}\right], \quad \text { for all } i, j=1, \ldots, L
$$

then (23) implies that

$$
X_{11}^{i j}=\left(X_{11}^{i j}\right)^{\top}=-X_{22}^{j i}, \quad\left(X_{12}^{i j}\right)^{\top}=-X_{12}^{j i} .
$$

Hence, the set $\left\{X^{i j} \in \mathbb{R}_{S}^{2 n_{G}^{i j}} \mid\right.$ (23) holds for all $i, j=$ $1, \ldots, L\}$ is parametrized by the two sets [2]

$$
\begin{aligned}
& \left\{X_{11}^{i j} \in \mathbb{R}_{S}^{n_{G}^{i j}} \mid i, j \in \mathbb{N}_{[1, L]}\right\}, \\
& \left\{X_{12}^{i j} \in \mathbb{R}_{G}^{n_{G}^{i j} \times n_{G}^{i j}} \mid i, j \in \mathbb{N}_{[1, L]}, j \leq i\right\} .
\end{aligned}
$$

Note that $X^{i j}$ is defined for all $i, j$ but has dimension zero if $j \notin \mathcal{N}^{i}$.

\section{Open loop analysis}

This section aims at deriving LMI feasibility conditions that guarantee a distributed dynamical system $G_{\Delta}$ to be well-posed, stable and with robust performance of level $\gamma$. We consider the uncontrolled system, which means that $u^{i}=0$ and $y^{i}$ is disregarded in all control channels $i=1, \ldots, L$.

Theorem 1. Let $G_{\Delta}$ be an uncertain distributed system with subsystems admitting realization (1)-(2) with property (12). Then $G_{\Delta}$ is well-posed, stable and achieves robust performance $\gamma$ for all inputs $d^{i} \in \ell_{2}^{n_{d}^{i}}$ if for all $i, j \in \mathbb{N}_{[1, L]}$ there exist matrices $X_{T}^{i} \in \mathbb{R}_{S}^{m^{i}}, D_{\Delta}^{i} \in \mathbb{R}_{S}^{2 n_{\Delta}^{i}}, X_{11}^{i j} \in \mathbb{R}_{S}^{n_{G}^{i j}}$ and for

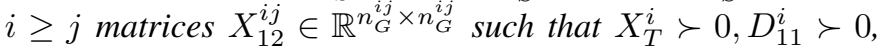

$D_{22}^{i} \prec 0$ and for all uncertainties $\Delta^{i} \in \Delta^{i}$ and $k \geq 0$ there holds

$$
\begin{array}{r}
\left(T^{i}\right)^{\top} M^{i} T^{i} \prec 0, \\
{\left[\begin{array}{c}
q^{i}(k) \\
\Delta^{i}\left(q^{i}(k)\right)
\end{array}\right]^{\top}\left[\begin{array}{cc}
D_{11}^{i} & D_{12}^{i} \\
\left(D_{12}^{i}\right)^{\top} & D_{22}^{i}
\end{array}\right]\left[\begin{array}{c}
q^{i}(k) \\
\Delta^{i}\left(q^{i}(k)\right)
\end{array}\right] \geq 0,}
\end{array}
$$

with

$$
\begin{aligned}
T^{i}:= & {\left[\begin{array}{cccc}
I & 0 & 0 & 0 \\
A_{x x}^{i} & A_{x v}^{i} & B_{x p}^{i} & B_{x d}^{i} \\
\hline A_{w x}^{i} & A_{w v}^{i} & B_{w p}^{i} & B_{w d}^{i} \\
0 & I & 0 & 0 \\
\hline C_{q x}^{i} & C_{q v}^{i} & D_{q p}^{i} & D_{q d}^{i} \\
0 & 0 & I & 0 \\
\hline C_{z x}^{i} & C_{z v}^{i} & D_{z p}^{i} & D_{z d}^{i} \\
0 & 0 & 0 & I
\end{array}\right], } \\
M^{i}:= & \operatorname{diag}\left(\left[\begin{array}{cc}
-X_{T}^{i} & 0 \\
0 & X_{T}^{i}
\end{array}\right],\left[\begin{array}{cc}
Z_{11}^{i} & Z_{12}^{i} \\
\left(Z_{12}^{i}\right)^{\top} & Z_{22}^{i}
\end{array}\right],\right. \\
& {\left.\left[\begin{array}{cc}
D_{11}^{i} & D_{12}^{i} \\
\left(D_{12}^{i}\right)^{\top} & D_{22}^{i}
\end{array}\right],\left[\begin{array}{cc}
\frac{1}{\gamma} I & 0 \\
0 & -\gamma I
\end{array}\right]\right), }
\end{aligned}
$$

and

$$
\begin{aligned}
& Z_{11}^{i}:=-\underset{j \in \mathbb{N}_{[1, L]}}{\operatorname{diag}} X_{11}^{i j}, \quad Z_{22}^{i}:=\underset{j \in \mathbb{N}_{[1, L]}}{\operatorname{diag}} X_{11}^{j i}, \\
& Z_{12}^{i}:=\operatorname{diag}\left(-\underset{j \in \mathbb{N}_{[1, i]}}{\operatorname{diag}} X_{12}^{i j}, \underset{j \in \mathbb{N}_{(i, L]}}{\operatorname{diag}}\left(X_{12}^{j i}\right)^{\top}\right) .
\end{aligned}
$$

Proof: Note that (27) has a block diagonal structure where the blocks relate to the state, interconnection, uncertainty and performance channels, respectively.

Suppose the conditions hold. Define $V^{i}\left(x^{i}\right)$ as in (20) and let $V(x):=\sum_{i=1}^{L} V^{i}\left(x^{i}\right)$ with $x=\left[x^{1}, \ldots, x^{L}\right]^{\top}$ a column stacked vector with all states of the subsystems. Pre- and postmultiply (27) with $\left[\left(x^{i}\right)^{\top}(k),\left(v^{i}\right)^{\top}(k),\left(p^{i}\right)^{\top}(k),\left(d^{i}\right)^{\top}(k)\right]$ and its transpose to infer that

$$
V^{i}\left(x^{i}(k+1)\right)-V^{i}\left(x^{i}(k)\right)<P^{i}(k)+U^{i}(k)+S^{i}(k) .
$$

Hence, each subsystem is strictly dissipative with respect to the aggregated supply function $\Phi^{i}(k)$ as defined in Section IV-A. Summing (32) over $i$ yields

$$
\begin{aligned}
V(x(k+1))-V(x(k))< & \sum_{i=1}^{L} P^{i}\left(v^{i}, w^{i}\right)+\sum_{i=1}^{L} U^{i}\left(p^{i}, q^{i}\right) \\
& +\sum_{i=1}^{L} S^{i}\left(d^{i}, z^{i}\right) .
\end{aligned}
$$

Since $\sum_{i=1}^{L} P^{i}\left(v^{i}, w^{i}\right)=0$ because of (23) and $\sum_{i=1}^{L} U^{i}\left(p^{i}, q^{i}\right) \leq 0$ by (18), (21) and (28), we get

$$
V(x(k+1))-V(x(k))<\sum_{i=1}^{L} S^{i}\left(d^{i}, z^{i}\right) .
$$

To prove stability, let $d^{i}=0$. Then $\sum_{i=1}^{L} S^{i}\left(0, z^{i}\right) \leq 0$ by (19) and we infer that

$$
V(x(k+1))-V(x(k))<0 .
$$


Since $X_{T}^{i} \succ 0$ it follows that $V(x(k))>0$. Hence, $V$ is a quadratic Lyapunov function for the uncertain distributed system, rendering it stable for all possible uncertainties $\Delta^{i} \in$ $\boldsymbol{\Delta}^{i}$. By construction of $V(x)$, we have that

$$
\begin{aligned}
\lambda_{\min }\left(\operatorname{diag}_{i \in \mathbb{N}_{[1, L]}} X_{T}^{i}\right)\|x\|_{2}^{2} \leq V(x) & =x^{\top}\left(\underset{i \in \mathbb{N}_{[1, L]}}{\operatorname{diag}} X_{T}^{i}\right) x \\
& \leq \lambda_{\max }\left(\underset{i \in \mathbb{N}_{[1, L]}}{\operatorname{diag}} X_{T}^{i}\right)\|x\|_{2}^{2}
\end{aligned}
$$

which shows that the uncertain system is globally exponentially stable in Lyapunov sense.

To prove robust performance, suppose that $d \neq 0$ and set $x(0)=0$. By summing over all time instants $k$ as defined in (15), (34) becomes

$$
V(x(M))-V(0)<\gamma\|d\|_{2}^{2}-\frac{1}{\gamma}\|z\|_{2}^{2}
$$

Since $V(0)=0$ and $V(x)>0$ for $x \neq 0$, we get

$$
\|z\|_{2}^{2}<\gamma^{2}\|d\|_{2}^{2}
$$

from which we infer that $\left\|G_{\Delta}\right\|_{2,2}<\gamma$ is a robust performance bound for the system.

Since $\Delta^{i}$ consists of stable LTI systems, the inequality (28) can equivalently be interpreted in the frequency domain. That is, in the Fourier domain the relation $p^{i}=\Delta^{i}\left(q^{i}\right)$ is equivalently represented by the product $\hat{p}^{i}=\widehat{\Delta}^{i} \hat{q}^{i}$ where $\widehat{\Delta}^{i}$ is the frequency response of $\Delta^{i}$. Similarly, the uncertainty set $\widehat{\Delta^{i}}$ of Fourier transformed elements in $\Delta^{i}$ then consists of all complex-valued matrices $\Delta^{i}(j \omega), \omega \in \mathbb{R}$ and $\Delta^{i} \in \boldsymbol{\Delta}^{i}$. Throughout, we identify $\widehat{\Delta}^{i}$ with $\Delta^{i}$ and $\widehat{\boldsymbol{\Delta}}^{i}$ with $\boldsymbol{\Delta}^{i}$. By doing so, (28) is equivalent to the LMI

$$
\left[\begin{array}{c}
I \\
\Delta^{i}
\end{array}\right]^{\top}\left[\begin{array}{cc}
D_{11}^{i} & D_{12}^{i} \\
\left(D_{12}^{i}\right)^{\top} & D_{22}^{i}
\end{array}\right]\left[\begin{array}{c}
I \\
\Delta^{i}
\end{array}\right] \succeq 0 .
$$

where $\Delta^{i}$ is a complex-valued matrix in $\Delta^{i}$. The result therefore gives LMI conditions for robust stability and performance for any given uncertain distributed system. Thus Theorem 1 yields a solution to Problem 1 for the open loop case. We emphasize that matrices $X_{11}^{i j}$ and $X_{12}^{i j}$ as defined in (26) and variable $\gamma$ are shared between subsystems. The feasibility test is therefore not decomposable and needs to be solved in a centralized way.

\section{Closed loop analysis}

When applied to closed loop systems as defined in Section II-D, Theorem 1 provides the following robust stability and performance conditions.

Proposition 1. Let $\left(G_{\Delta}\right)_{C}$ be an uncertain distributed system with subsystems admitting the realization (4)-(5) with property (12)-(13). Then, the system is well-posed, stable and achieves robust performance $\gamma$ for all inputs $d^{i} \in \ell_{2}^{n_{d}^{i}}$ if $\forall i \in \mathbb{N}_{[1, L]}$ there exist matrices $\left(X_{T}^{i}\right)_{C} \in \mathbb{R}_{S}^{2 m^{i}}, Z_{C}^{i} \in \mathbb{R}_{S}^{2 n_{C}^{i}}$ and $D_{\Delta}^{i} \in$ $\mathbb{R}_{S}^{2 n_{\Delta}^{i}}$, such that $\left(X_{T}^{i}\right)_{C} \succ 0, D_{11}^{i} \succ 0, D_{22}^{i} \prec 0$ and for all
$\Delta^{i} \in \boldsymbol{\Delta}^{i}$

$$
\begin{aligned}
\left(T_{C}^{i}\right)^{\top} M_{C}^{i} T_{C}^{i} & \prec 0, \\
{\left[\begin{array}{c}
I \\
\Delta^{i}
\end{array}\right]^{\top}\left[\begin{array}{cc}
D_{11}^{i} & D_{12}^{i} \\
\left(D_{12}^{i}\right)^{\top} & D_{22}^{i}
\end{array}\right]\left[\begin{array}{c}
I \\
\Delta^{i}
\end{array}\right] } & \succeq 0,
\end{aligned}
$$

with

$$
\begin{aligned}
& T_{C}^{i}:=\left[\begin{array}{cccc}
I & 0 & 0 & 0 \\
\left(A_{x x}^{i}\right)_{C} & \left(A_{x v}^{i}\right)_{C} & \left(B_{x p}^{i}\right)_{C} & \left(B_{x d}^{i}\right)_{C} \\
\hline\left(A_{w x}^{i}\right)_{C} & \left(A_{w v}^{i}\right)_{C} & \left(B_{w p}^{i}\right)_{C} & \left(B_{w d}^{i}\right)_{C} \\
0 & I & 0 & 0 \\
\left(C_{q x}^{i}\right)_{C} & \left(C_{q v}^{i}\right)_{C} & \left(D_{q p}^{i}\right)_{C} & \left(D_{q d}^{i}\right)_{C} \\
0 & 0 & I & 0 \\
\hline\left(C_{z x}^{i}\right)_{C} & \left(C_{z v}^{i}\right)_{C} & \left(D_{z p}^{i}\right)_{C} & \left(D_{z d}^{i}\right)_{C} \\
0 & 0 & 0 & I
\end{array}\right], \\
& M_{C}^{i}:=\operatorname{diag}\left(\left[\begin{array}{cc}
-\left(X_{T}^{i}\right)_{C} & 0 \\
0 & \left(X_{T}^{i}\right)_{C}
\end{array}\right],\left[\begin{array}{ll}
\left(Z_{11}^{i}\right)_{C} & \left(Z_{12}^{i}\right)_{C} \\
\left(Z_{12}^{i}\right)_{C} & \left(Z_{22}^{i}\right)_{C}
\end{array}\right]\right. \text {, } \\
& \left.\left[\begin{array}{cc}
D_{11}^{i} & D_{12}^{i} \\
\left(D_{12}^{i}\right)^{\top} & D_{22}^{i}
\end{array}\right],\left[\begin{array}{cc}
\frac{1}{\gamma} I & 0 \\
0 & -\gamma I
\end{array}\right]\right)
\end{aligned}
$$

and with $\left(Z_{11}^{i}\right)_{C}$ partitioned as

$$
\left(Z_{11}^{i}\right)_{C}:=\left[\begin{array}{cc}
\left(Z_{11}^{i}\right)_{G} & \left(Z_{11}^{i}\right)_{G K} \\
\left(Z_{11}^{i}\right)_{G K}^{\top} & \left(Z_{11}^{i}\right)_{K}
\end{array}\right],
$$

and $\left(Z_{12}^{i}\right)_{C},\left(Z_{22}^{i}\right)_{C}$ and $\left(X_{T}^{i}\right)_{C}$ defined analogously.

The above proposition is a direct extension of Theorem 1, where the Lyapunov matrices $X_{T}^{i}$ and interconnection matrices $Z^{i}$ are extended to their closed loop counterparts $\left(X_{T}^{i}\right)_{C}$ and $\left(Z^{i}\right)_{C}$. The matrices $\left(Z^{i}\right)_{G},\left(Z^{i}\right)_{K}$ and $\left(Z^{i}\right)_{G K}$ model the interconnections between plants, controllers, and their cross-terms. The construction of these matrices from the individual supply function matrices $\left(X^{i j}\right)_{G}$ (and $\left(X^{i j}\right)_{K}$ and $\left(X^{i j}\right)_{G K}$, respectively) on the interconnection channels, which are defined analogous to (24) and parameterized as in (26), is straightforward but somewhat tedious, and the reader is referred to [2, Proposition 2] for details. Proposition 1 provides an LMI feasibility test for a distributed controlled system to be well-posed, robustly stable and with robust performance. Thus it yields a solution to Problem 1 for the closed loop case.

\section{SYNTHESIS}

In this section we propose a synthesis algorithm to solve Problem 2. The algorithm is based on Theorem 1 and the approach will be twofold. First, we derive sufficient conditions for the existence of a distributed controller satisfying Proposition 1. Then the controller is constructed.

\section{A. Controller existence}

The conditions in Proposition 1 yield non-linear matrix inequalities when solving for the unknown matrices in $M^{i}$ and the unknown controller parameters $\Theta^{i}$. To get LMI conditions, we eliminate the controller parameters $\Theta^{i}$ from the closed loop conditions using the following lemma from [16]. 
Lemma 1 (Elimination lemma). Let $M$ be a symmetric matrix with in $(M)=\left(a_{-}, 0, a_{+}\right)$and matrix $\Pi^{i} \in \mathbb{R}^{a_{+} \times a_{-}}$. Then the matrix inequality

$$
\left[\begin{array}{c}
I \\
\Pi+\Psi^{\top} \Theta \Phi
\end{array}\right]^{\top} M\left[\begin{array}{c}
I \\
\Pi+\Psi^{\top} \Theta \Phi
\end{array}\right] \prec 0
$$

in the unstructured unknown $\Theta$ has a solution if and only if

$$
\begin{array}{r}
\Phi_{\perp}^{\top}\left[\begin{array}{c}
I \\
\Pi
\end{array}\right]^{\top} M\left[\begin{array}{c}
I \\
\Pi
\end{array}\right] \Phi_{\perp} \prec 0, \\
\Psi_{\perp}^{\top}\left[\begin{array}{c}
-\Pi^{\top} \\
I
\end{array}\right]^{\top} M^{-1}\left[\begin{array}{c}
-\Pi^{\top} \\
I
\end{array}\right] \Psi_{\perp} \succ 0 .
\end{array}
$$

Here, $\Phi_{\perp}$ and $\Psi_{\perp}$ are matrices whose columns are minimal spanning sets of the nullspace of $\Phi$ and $\Psi$, respectively. Furthermore, if a solution exists, $\Theta$ can be explicitly reconstructed from the matrices in (45) and (46).

This lemma provides LMI feasibility conditions for the existence of a distributed controller and the possibility to explicitly construct such a the controller if it exists. The LMI conditions for existence of a controller are collected in the following theorem.

Theorem 2. There exist matrices such that the conditions of Proposition 1 are satisfied with $n_{K}^{i j}=3 n_{G}^{i j}$ if and only if for all $i \in \mathbb{N}_{[1, L]}$ there exist parameterized symmetric matrices $M_{G}^{i}, \tilde{M}_{G}^{i} \in \mathbb{R}_{S}^{2\left(m^{i}+n_{G}^{i}+n_{\Delta}^{i}\right)+n_{d}^{i}+n_{z}^{i}}$, as defined below, such that $D_{11}^{i} \succ 0, D_{22}^{i} \prec 0,\left(X_{T}^{i}\right)_{G} \succ 0,\left(Y_{T}^{i}\right)_{G} \succ 0$, and for all $\Delta^{i} \in \boldsymbol{\Delta}^{i}$,

$$
\begin{aligned}
\left(\Phi_{G}^{i}\right)_{\perp}^{\top}\left(T^{i}\right)^{\top} M_{G}^{i} T^{i}\left(\Phi_{G}^{i}\right)_{\perp} & \prec 0, \\
\left(\Psi_{G}^{i}\right)_{\perp}^{\top}\left(T_{\perp}^{i}\right)^{\top} \tilde{M}_{G}^{i} T_{\perp}^{i}\left(\Psi_{G}^{i}\right)_{\perp} & \succ 0, \\
{\left[\begin{array}{cc}
\left(X_{T}^{i}\right)_{G} & I \\
I & \left(Y_{T}^{i}\right)_{G}
\end{array}\right] } & \succeq 0, \\
{\left[\begin{array}{c}
I \\
\Delta^{i}
\end{array}\right]^{\top}\left[\begin{array}{cc}
D_{11}^{i} & D_{12}^{i} \\
\left(D_{12}^{i}\right)^{\top} & D_{22}^{i}
\end{array}\right]\left[\begin{array}{c}
I \\
\Delta^{i}
\end{array}\right] } & \succeq 0 .
\end{aligned}
$$

Here,

$\left(\Phi_{G}^{i}\right)_{\perp}^{\top}$ spans the nullspace of $\left[C_{y x}^{i} C_{y v}^{i} D_{y p}^{i} D_{y d}^{i}\right]$,

$\left(\Psi_{G}^{i}\right)_{\perp}^{\top}$ spans the nullspace of $\left[\left(B_{x u}^{i}\right)^{\top}\left(B_{w u}^{i}\right)^{\top}\left(D_{q u}^{i}\right)^{\top}\left(D_{z u}^{i}\right)^{\top}\right]$,

$$
T^{i}:=\left[\begin{array}{cccc}
I & 0 & 0 & 0 \\
A_{x x}^{i} & A_{x v}^{i} & B_{x p}^{i} & B_{x d}^{i} \\
A_{w x}^{i} & A_{w v}^{i} & B_{w p}^{i} & B_{w d}^{i} \\
0 & I & 0 & 0 \\
\hline C_{q x}^{i} & C_{q v}^{i} & D_{q p}^{i} & D_{q d}^{i} \\
0 & 0 & I & 0 \\
C_{z x}^{i} & C_{z v}^{i} & D_{z p}^{i} & D_{z d}^{i} \\
0 & 0 & 0 & I
\end{array}\right],
$$

$$
\begin{gathered}
M_{G}^{i}:=\operatorname{diag}\left(\begin{array}{cc}
-\left(X_{T}^{i}\right)_{G} & 0 \\
0 & \left(X_{T}^{i}\right)_{G}
\end{array}\right],\left[\begin{array}{cc}
\left(Z_{11}^{i}\right)_{G} & \left(Z_{12}^{i}\right)_{G} \\
\left(Z_{12}^{i}\right)_{G} & \left(Z_{22}^{i}\right)_{G}
\end{array}\right], \\
\left.\left[\begin{array}{cc}
D_{11}^{i} & D_{12}^{i} \\
\left(D_{12}^{i}\right)^{\top} & D_{22}^{i}
\end{array}\right],\left[\begin{array}{cc}
\frac{1}{\gamma} I & 0 \\
0 & -\gamma I
\end{array}\right]\right), \\
\tilde{M}_{G}^{i}:=\operatorname{diag}\left(\left[\begin{array}{cc}
-\left(Y_{T}^{i}\right)_{G} & 0 \\
0 & \left(Y_{T}^{i}\right)_{G}
\end{array}\right],\left[\begin{array}{ll}
\left(\tilde{Z}_{11}^{i}\right)_{G} & \left(\tilde{Z}_{12}^{i}\right)_{G} \\
\left(\tilde{Z}_{12}^{i}\right)_{G} & \left(\tilde{Z}_{22}^{i}\right)_{G}
\end{array}\right],\right. \\
\left.\left[\begin{array}{cc}
D_{11}^{i} & D_{12}^{i} \\
\left(D_{12}^{i}\right)^{\top} & D_{22}^{i}
\end{array}\right]^{-1},\left[\begin{array}{cc}
\gamma I & 0 \\
0 & -\frac{1}{\gamma} I
\end{array}\right]\right),
\end{gathered}
$$

with further parameterization by supply function matrices $\left(X^{i j}\right)_{G}$ and $\left(Y^{i j}\right)_{G}$ which are defined analogous to $(24)$ and parameterized as in (26), as

$\left(Z_{11}^{i}\right)_{G}:=-\operatorname{diag}_{j \in \mathbb{N}_{[1, L]}}\left(X_{11}^{i j}\right)_{G}, \quad\left(\tilde{Z}_{11}^{i}\right)_{G}:=-\operatorname{diag}_{j \in \mathbb{N}_{[1, L]}}\left(Y_{11}^{i j}\right)_{G}$, and $\left(Z_{12}^{i}\right)_{G},\left(Z_{22}^{i}\right)_{G},\left(\tilde{Z}_{12}^{i}\right)_{G},\left(\tilde{Z}_{22}^{i}\right)_{G}$ are defined analogously.

Proof: (Only if:) Consider Proposition 1 and note that (39) is satisfied if and only if

$$
T^{\top} M T \prec 0 \text { for } T=\underset{i \in \mathbb{N}_{[1, L]}}{\operatorname{diag}}\left(T_{C}^{i}\right), M=\underset{i \in \mathbb{N}_{[1, L]}}{\operatorname{diag}}\left(M_{C}^{i}\right)
$$

To apply Lemma 1 to (39), we consider the inertia of $M$, which is given by

$$
\begin{aligned}
\operatorname{in}(M)= & \sum_{i=1}^{L} i n\left(\left[\begin{array}{cc}
-\left(X_{T}^{i}\right)_{C} & 0 \\
0 & \left(X_{T}^{i}\right)_{C}
\end{array}\right]\right)+\sum_{i, j=1}^{L} i n\left(-\left(X^{i j}\right)_{C}\right) \\
& +\sum_{i=1}^{L} i n\left(D_{\Delta}^{i}\right)+\sum_{i=1}^{L}\left(n_{d}^{i}, 0, n_{z}^{i}\right) .
\end{aligned}
$$

Since $\left(X_{T}^{i}\right)_{C} \succ 0$ and $\operatorname{in}\left(D_{\Delta}^{i}\right)=\operatorname{in}\left(D_{11}^{i}\right)+i n\left(D_{22}^{i}-\right.$ $\left.\left(D_{12}^{i}\right)^{\top}\left(D_{11}^{i}\right)^{-1} D_{12}^{i}\right)$ with $D_{11}^{i} \succ 0, D_{22}^{i} \prec 0$ and using the interconnection multipliers in (26), this is equal to

$$
\begin{array}{r}
i n(M)=\left(\sum_{i=1}^{L}\left(2 m^{i}+n_{C}^{i}+n_{\Delta}^{i}+n_{d}^{i}\right), 0,\right. \\
\left.\sum_{i=1}^{L}\left(2 m^{i}+n_{C}^{i}+n_{\Delta}^{i}+n_{z}^{i}\right)\right) .
\end{array}
$$

By construction, the inertia requirement on $M$ holds, and (51) can be brought into the form of Lemma 1 through matrix permutation. By eliminating the controller parameters, permuting the lines and columns back to the block-diagonal representation and selecting only the rows and columns associated with interconnection and Lyapunov matrices $(\cdot)_{G}$, we arrive at (47)-(48) with $M_{G}^{i}$ and $\tilde{M}_{G}^{i}$ truncated versions of $M_{C}^{i}$ and $\left(M_{C}^{i}\right)^{-1}$, respectively.

(If:) The sufficiency part of the proof follows along the lines of [6, Theorem 2]. For $n_{K}^{i j}=3 n_{G}^{i j}$, which is a sufficient condition for the inertia condition to hold, and (49) the primal and dual inequalities can be used to extend the interconnection and Lyapunov matrices to full-block matrices that satisfy Proposition 1.

The result of Theorem 2 does not define a convex optimization problem in all variables. Applying the elimination lemma on the analysis equations of Proposition 1 removes the controller parameters from the equations, thus removing the non-linearity obtained from multiplying controller parameters and multipliers. However, (47) and (48) yield a set of LMIs in both $D_{\Delta}^{i}$ and $\left(D_{\Delta}^{i}\right)^{-1}$ which renders the combined set of equations non-convex. A remedy to this problem is suggested in Section V-C. For the nominal controller synthesis problem, where no uncertainty is considered, Theorem 2 provides a convex problem that can be solved directly by taking all matrices corresponding to uncertainty to be of dimension zero. 


\section{B. Controller construction}

Using Lemma 1, a controller can be inferred from the feasibility test of Theorem 2. The solutions of the primal and dual inequalities can be used to extend the interconnection and Lyapunov matrices to full-block matrices satisfying Proposition 1. For details on this construction we refer to [2], [5], [16]. The synthesized controller achieves well-posedness for the entire controlled system if it satisfies (13).

\section{D-K synthesis algorithm}

Both Proposition 1 and Theorem 2 give non-convex conditions for controller synthesis. However, for given (nonsingular) uncertainty scalings $D_{\Delta}^{i}$, Theorem 2 gives convex conditions while for a given controller $K$, Proposition 1 gives convex conditions. A D-K iterative algorithm will therefore be proposed that alternates between these two scenarios to find a solution to Problem 2.

Algorithm 1. Initialisation: $j=0, j_{\max } \in \mathbb{N}, \epsilon>0$.

1) Initial K-step: Apply Theorem 2 for the nominal system $G_{0}$ and with $D_{\Delta}^{i}$ of dimension zero for all $i$ to find an initial $\gamma_{K, 0}$ and a distributed controller $K_{0}$. Set $j=1$.

2) Initial D-step: Apply Proposition 1 for the closed loop uncertain system $G_{\Delta}$ controlled by $K_{0}$ to find matrices $\left(D_{\Delta}^{i}\right)_{1}$, $i=1, \ldots, L$, with minimal performance bound $\gamma_{D, 1}$.

3) D-K iteration:

a) K-step: Apply Theorem 2 for the nominal system $G_{0}$ and $\left(D_{\Delta}^{i}\right)_{j}$ for all $i$ from the previous $D$-step to find a robust distributed controller $K_{j}$ obtaining performance $\gamma_{K, j}$. Set $j=j+1$.

b) D-step: Apply Proposition 1 for the closed loop uncertain system $G_{\Delta}$ controlled by $K_{j-1}$ to find $\left(D_{\Delta}^{i}\right)_{j}$, $i=1, \ldots, L$, with minimal performance bound $\gamma_{D, j}$.

c) Termination: End the iteration if the decrease of $\gamma_{K, j}$ is smaller than $\epsilon$ or $j>j_{\max }$.

The maximum number of iterations $j_{\max }$ and the convergence precision $\epsilon$ should be chosen sufficiently large and small, respectively. Key properties of this algorithm are given in the following result.

Theorem 3. Let $G_{\Delta}$ be an uncertain distributed system. Suppose there exists $K_{0}, \gamma_{D, 1}$ and $\left(D_{\Delta}^{i}\right)_{1}$ for all $i$ as defined in Algorithm 1. Then the $D-K$ iteration runs and the solution to Algorithm 1 has the following properties:

1) $\gamma_{K, j} \leq \gamma_{K, j-1}$ for all $1<j \leq j_{\max }$.

2) Problem 2 is solvable with robust performance bound $\gamma=\lim _{j \rightarrow \infty} \gamma_{K, j}$ and $\gamma=\gamma_{K, j_{\max }}$.

Proof: $K_{0}$ defines a well-posed and stable nominally controlled system $\left(G_{0}\right)_{C}$ with finite $H_{\infty}$ norm $\gamma_{K, 0}$. The initial $D$-step provides scalings $\left(D_{\Delta}^{i}\right)_{1}$ such that $\left(G_{\Delta}\right)_{C}$ is, in addition, robustly stable against uncertainties $\Delta^{i} \in \Delta^{i}$ with robust performance bound $\gamma_{D, 1}$. To prove item 1 , consider the $j$ th iteration of step 3 a with uncertainty (18) defined by the scalings $\left(D_{\Delta}^{i}\right)_{j-1}$. Minimize $\gamma$ subject to feasibility of (47)-(50) to infer a new $\gamma_{K, j}$ and a controller $K_{j}$. From the $(j-1)$ st iteration we established robust performance at level $\gamma_{D, j-1}$ with controller $K_{j-1}$. Hence, in the $j$ th iteration, (47)-(50) is feasible with $\gamma=\gamma_{K, j}=\gamma_{D, j-1}$. Minimizing $\gamma$ subject to (47)-(50) therefore gives $\gamma_{K, j} \leq \gamma_{D, j-1}$. By a similar argument, we infer from the $D$-step that $\gamma_{D, j} \leq \gamma_{K, j}$. Combining the two arguments shows that $\gamma_{K, j} \leq \gamma_{K, j-1}$ for any $j>1$. The algorithm therefore yields a non-increasing sequence of robust performance levels $\gamma_{K, j}$. Taking the limit shows that Problem 2 is solvable with $\gamma=\lim _{j \rightarrow \infty} \gamma_{K, j}$.

Hence, every robust controller $K_{j}$ inferred from step $3 \mathrm{a}$ solves Problem 2. Theorem 3 proves monotonic convergence of the performance bound $\gamma$ provided the algorithm passes step 2 . Every D-K iteration provides a robust performance bound that improves the one from the previous iteration. The algorithm does converge, but not necessarily to a globally optimal robust performance level. The rate of convergence will depend on the system dynamics and uncertainty. Also note that step 1 gives a solution to Problem 2 for the case without uncertainty, i.e. for the nominal system.

The number of decision variables in this optimization provides an indication of the numerical complexity of Algorithm 1. The matrices $X_{T}^{i},\left(X_{T}^{i}\right)_{K},\left(X_{T}^{i}\right)_{G K}$ and $\left(X_{T}^{i}\right)_{C}$, corresponding to the state, contain $\left(\left(m^{i}\right)^{2}+m^{i}\right) / 2,\left(\left(m^{i}\right)^{2}+\right.$ $\left.m^{i}\right) / 2,\left(m^{i}\right)^{2}$ and $2\left(m^{i}\right)^{2}+m^{i}$ unknown variables per subsystem $i$, respectively. The matrices $D_{\Delta}^{i}$, corresponding to the uncertainty, contain $2\left(n_{\Delta}^{i}\right)^{2}+n_{\Delta}^{i}$ unknown variables per subsystem $i$. For the interconnections, we have $L^{2}$ matrices $X_{11}^{i j}$ (for $\left.i, j=1, \ldots, L\right)$, each having $\left(\left(n_{G}^{i j}\right)^{2}+n_{G}^{i j}\right) / 2$ independent variables, or $\left(\left(n_{C}^{i j}\right)^{2}+n_{C}^{i j}\right) / 2$ for the closed loop variables $\left(X_{11}^{i j}\right)_{C}$, and we have $\left(L^{2}+L\right) / 2$ matrices $X_{12}^{i j}$ (for $1 \leq j \leq i \leq L)$, each having $\left(n_{G}^{i j}\right)^{2}$ unknown variables, or $\left(n_{C}^{i j}\right)^{2}$ for the closed loop variables $\left(X_{12}^{i j}\right)_{C}$. Recall that $n_{C}^{i j}=n_{G}^{i j}+n_{K}^{i j}$ with $n_{K}^{i j}=3 n_{G}^{i j}$.

\section{SimUlation AND RESUlTS}

To illustrate the computational feasibility of the presented framework, the synthesis routine has been implemented and applied to a power network as an example. For a proper comparison, a centralized $\mathcal{H}_{\infty}$ optimal controller for the nominal system, a distributed $\mathcal{H}_{\infty}$ controller for the nominal system and a distributed robust $\mathcal{H}_{\infty}$ controller have been derived following the procedure outlined in this paper. All computations have been carried out in Matlab and by using freely available software [17] and [18].

\section{A. Power network simulations}

Consider the four generator model, as described by [6]. This model consists of four control areas that are interconnected by tie lines. The tie line dynamics are incorporated in the plant model of the preceding control area as shown in Fig. 1. Changes in load at the control areas are considered as the

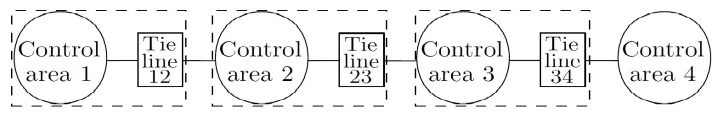

Fig. 1. [6, Fig. 5] Four generator model. The tie line dynamics between area $i$ and $j$ are lumped into the $i$ th area dynamics for $i<j$.

disturbance input $d$. The performance is measured by the 
performance output $z$ that consists of the tie line power from a neighboring area, the frequency deviation and the reference power from the controller, for each control area. The controller aims to regulate the tie line power and frequency deviation as close as possible to 0. Loop shaping filters, which are integrated into the plant model, are used to do tune the frequency characteristics of the controlled system. The same uncertainties as in the continuous time case have been introduced to produce the uncertain plant. The continuous time model is discretized by a zero-order hold $(\mathrm{ZOH})$ method with a sampling time of $0.1 \mathrm{sec}$. A robust distributed controller of total state size $m_{K}=m_{G}=44$ is obtained by Algorithm 1 . In Tab. I the resulting $\mathcal{H}_{\infty}$-norm of the closed loop transfer functions $d \rightarrow z$ is shown for each type of controller. The value of the performance bound as calculated by the controller synthesis algorithm $\gamma_{K}$ is given, as well as the results when the controller is connected to a nominal plant $\left\|\left(G_{0}\right)_{C}\right\|_{\infty}$ and a sample perturbed plant $\left\|\left(G_{\Delta}\right)_{C}\right\|_{\infty}$.

TABLE I. PERFORMANCE BOUNDS OF THE RESULTING CLOSED LOOP SYSTEMS FOR THE POWER NETWORK

\begin{tabular}{llll} 
Controller & $\gamma_{K}$ & $\left\|\left(G_{0}\right)_{C}\right\|_{\infty}$ & $\left\|\left(G_{\Delta}\right)_{C}\right\|_{\infty}$ \\
\hline Nominal Centralized $K_{\mathcal{H}}$ & $5.69 \mathrm{e}+00$ & $3.49 \mathrm{e}+00$ & $3.27 \mathrm{e}+00$ \\
Nominal Distributed $K$ & $3.28 \mathrm{e}+01$ & $1.91 \mathrm{e}+01$ & $1.85 \mathrm{e}+01$ \\
Robust Distributed $K$ & $4.99 \mathrm{e}+05$ & $2.49 \mathrm{e}+03$ & $2.39 \mathrm{e}+03$ \\
\hline
\end{tabular}

The results in Tab. I show that the robust controller achieves a significantly larger performance bound compared to the nominal and centralized controllers. When applied to the nominal system or a perturbed system the bound is reduced significantly, but there is still a large difference. Compared to the continuous time results by [6] a comparable observation can be made, although the bounds are significantly higher for the discrete time case. In Fig. 2 the maximum singular values
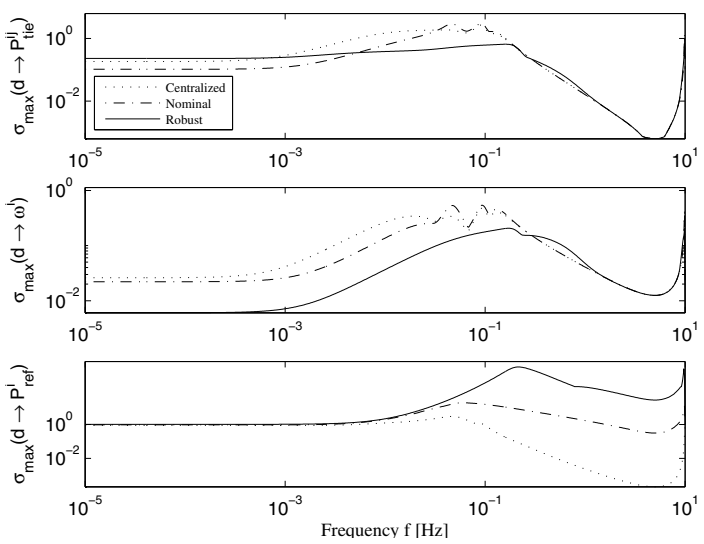

Fig. 2. Comparison of the maximum singular values with different control strategies, per performance channel.

of the transfer function mapping the disturbance inputs to the different performance channels are shown for each closed loop system. For each controller, similar maximum singular value curves can be seen for the tie-line power $P_{t i e}$ and frequency deviation $\omega$. This comes at the cost of a much larger reference power $P_{\text {ref }}$ which corresponds to a much larger control effort. It can be said that the same performance as in the centralized case is achieved for the tie-lines and frequency deviation at the cost of a larger control effort for the robust case. From the plots, it can be seen that, for example for the frequency deviation in lower frequencies, the robust controller outperforms the centralized one, but again at the cost of a larger control effort. As described in Section V-C the solution may not be a global optimum, therefore, a controller with lower bound may be found but with worse performance for the frequency deviation.

Time simulations of the closed loop systems are shown in which the disturbance input is taken as a stepwise load increase of $25 \%$ in area 2 at time $t=10 \mathrm{~s}$ and a $25 \%$ load decrease in area 3 at $t=20 \mathrm{~s}$. The time-domain simulation results are depicted in Fig. 3. The simulations show that the tie-line power
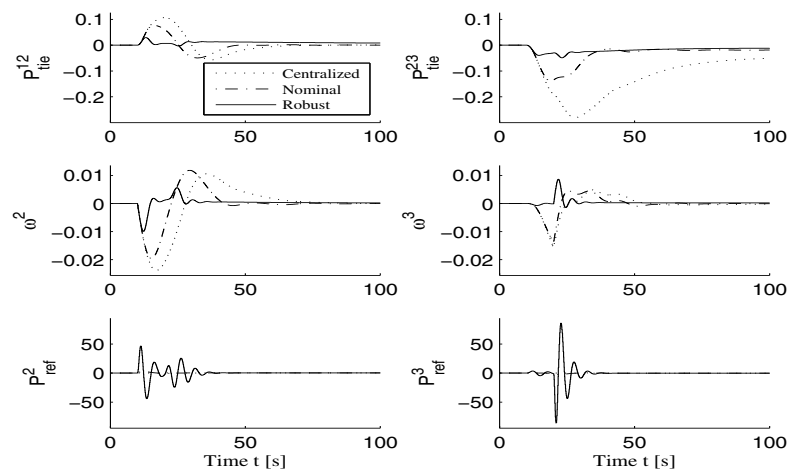

Fig. 3. Comparison of time domain behavior of different controllers on the nominal system.

and frequency deviations can be kept small at the cost of a large control effort. In particular, these results show that, for the power network, the discrete time synthesis procedure presented in this paper provides a feasible solution for this network.

\section{CONCLUSIONS AND COMMENTS}

In this paper we presented a number of analysis and synthesis results for distributed robust $\mathcal{H}_{\infty}$ control of interconnected discrete time dynamical systems. The main result involves a synthesis algorithm that is based on feasibility tests involving linear matrix inequalities (LMIs). With modern numerical tools in semi-definite programming the presented algorithm is computationally powerful and efficient. The algorithm resembles a so called $\mathrm{D}-\mathrm{K}$ type of iterative procedure that is known in $\mu$-controller synthesis. The resulting distributed controller renders an uncertain distributed network of interconnected discrete time LTI systems well-posed, robustly stable with a guaranteed robust $\mathcal{H}_{\infty}$ performance level $\gamma$. The synthesis of the communication channels among the controllers is part of the synthesis procedure. The algorithm is computationally tractable for moderate size networks. The paper also derives analysis tools to verify robust stability and robust performance of a given network in terms of LMI feasibility tests.

This work is inspired by earlier contributions in [2], [5], [6], [11], but the application of robust controller tools to discrete 
time systems is a novel feature of this work. In particular, the discrete time nature of the systems allow an investigation of communication delays that cannot be performed in the realm of finite dimensional continuous time systems. The application of this theory to systems with delays is therefor interesting in this respect.

A simulation is provided to illustrate the proper working of the proposed algorithm and yield a proof of concept.

The scope of the work is mainly limited by computer power. More specifically, by the number of decision variables that can be handled in the interior-point optimizations that solve the linear matrix inequalities that are derived for the analysis and controller synthesis in the main theorems of this paper. The presented methodology is based on dissipation considerations from which a synthesis procedure has been deduced that relies on a complete model of the network under consideration. That is, the distributed controllers are synthesized as the result of an efficient interior-point optimization that involves a complete model of the network. This may be viewed as a limitation of the presented methodology as it does not account for a distributed or decentralized synthesis. The complexity of the network, measured in terms of the state dimension of its constituent components therefore imposes a computational limitation to the given method. a partial remedy to this problem lies in the possibility to reduce the problem complexity by fixing interconnection variables, fixing supply functions in the communication channels or by fixing Lyapunov functions in the stability assessments. Currently, research is being conducted to achieve these complexity reductions in a computationally tractable way [19].

\section{ACKNOWLEDGEMENT}

The authors would like to thank dr. M. Lazar for constructive discussions on this research.

\section{REFERENCES}

[1] R. D'Andrea and G. Dullerud, "Distributed control design for spatially interconnected systems," Automatic Control, IEEE Transactions on, vol. 48, no. 9, pp. 1478-1495, 2003.

[2] C. Langbort, R. S. Chandra, and R. D'Andrea, "Distributed control design for systems interconnected over an arbitrary graph," Automatic Control, IEEE Transactions on, vol. 49, no. 9, pp. 1502-1519, 2004.

[3] G. Dullerud and R. D'Andrea, "Distributed control of heterogeneous systems," Automatic Control, IEEE Transactions on, vol. 49, no. 12, pp. 2113-2128, 2004.

[4] J. C. Willems, "Dissipative dynamical systems part I: General theory," Archive for Rational Mechanics and Analysis, vol. 45, pp. 321-351, Jan. 1972.

[5] G. W. Dekker, "Distributed H-infinity-based control of electrical power systems," Master's thesis, Eindhoven University of Technology, 2010.

[6] T. F. van der Els, "Robust distributed H-infinity control with an application to electrical power systems," Master's thesis, Eindhoven University of Technology, 2011.

[7] A. Jokic, T. F. Van der Els, and S. Weiland, "Robust distributed h-infinity control of electrical power systems," in American Control Conference (ACC), 2012, 2012, pp. 3637-3642

[8] R. Chandra, E. D'Andrea, and C. Langbort, "Distributed control design with robustness to small time delays," in American Control Conference, 2005. Proceedings of the 2005, 2005, pp. 4850-4855 vol. 7.
[9] R. D'Andrea and R. Chandra, "Control of spatially interconnected discrete-time systems," in Decision and Control, 2002, Proceedings of the 41st IEEE Conference on, vol. 1, 2002, pp. 240-245 vol.1.

[10] H. Li, Q. Wu, and D. Yu, "Distributed h-infinity; control of spatially interconnected systems with random communication packet losses," in Decision and Control, 2009 held jointly with the 2009 28th Chinese Control Conference. CDC/CCC 2009. Proceedings of the 48th IEEE Conference on, 2009, pp. 2046-2051.

[11] F. Wu, "Distributed control for interconnected linear parameterdependent systems," Control Theory and Applications, IEEE Proceedings -, vol. 150, no. 5, pp. 518-527, 2003.

[12] R. Diestel, Graph Theory, 4th ed., ser. Graduate Texts in Mathematics. Springer-Verlag, Heidelberg, July 2010, vol. 173.

[13] R. Redheffer, "On a certain linear fractional transformation," J. Math. Phys., vol. 39, pp. 269-286, 1960.

[14] M. Lazar, "Model predictive control of hybrid systems: Stability and robustness," Ph.D. dissertation, Eindhoven University of Technology, 2006.

[15] C. W. Scherer and S. Weiland, "Linear Matrix Inequalities in Control," 2005, Lecture Notes to DISC (Dutch Institute for Systems and Control) course Linear Matrix Inequalities in Control.

[16] C. W. Scherer, "LPV control and full block multipliers," Automatica, vol. 37, no. 3, pp. $361-375,2001$.

[17] J. Lofberg, "Yalmip : a toolbox for modeling and optimization in matlab," in Computer Aided Control Systems Design, 2004 IEEE International Symposium on, 2004, pp. 284-289.

[18] J. F. Sturm, "Using sedumi 1.02, a MATLAB toolbox for optimization over symmetric cones," Optimization Methods and Software, vol. 1112, pp. 625-653, 1999.

[19] R. V. Bobiti, R. H. Gielen, and M. Lazar, "Nonconservative and tractable stability tests for general linear interconnected systems with an application to power systems," in 4th IFAC Workshop on Distributed Estimation and Control in Networked Systems, NecSys 2013, 2013, pp. 152-159.

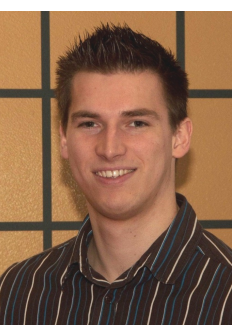

Eelco van Horssen received the M.Sc. degree (cum laude) in Electrical Engineering in 2013 from the Eindhoven University of Technology (TU/e), The Netherlands. Currently, he is with the Department of Mechanical Engineering of the TU/e pursuing a $\mathrm{Ph} . \mathrm{D}$. degree. His reseach interests include general theory of systems and control, distributed control, robust stability and performance, switched systems, networked and embedded control, dynamic programming, optimal control, and vision-based systems.

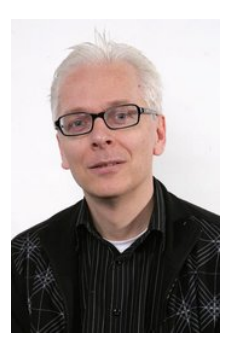

Siep Weiland received the M.Sc. (1986) and Ph.D. (1991) degrees in mathematics from the University of Groningen, The Netherlands. He was a Postdoctoral Research Associate at the Department of Electrical Engineering and Computer Engineering, Rice University, Houston, USA, from 1991 to 1992. Since 1992, he has been affiliated with Eindhoven University of Technology, Eindhoven, The Netherlands. $\mathrm{He}$ is a Full Professor at the same university with the Control Systems Group, Department of Electrical Engineering. His research interests are the general theory of systems and control, robust control, model approximation, modeling and control of spatial-temporal systems, identification, and model predictive control. 